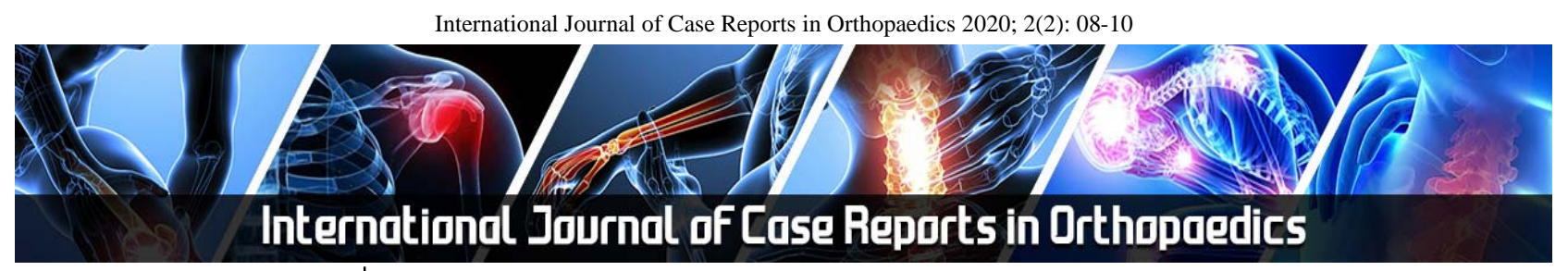

E-ISSN: 2707-8353

P-ISSN: 2707-8345

IJCRO 2020; 2(2): 08-10

Received: 06-05-2020

Accepted: 09-06-2020

Hoan Do Dang

Vice Dean of Doctors,

General Surgery Department,

Vietnam National Lung

Hospital, Vietnam

Trang Nguyen Khac

Dean of Doctors,

General Surgery Department,

Vietnam National Lung

Hospital, Vietnam

Nhung Nguyen Viet

Director of Vietnam National

Lung Hospital, Vietnam
Corresponding Author: Trang Nguyen Khac Dean of Doctors,

General Surgery Department, Vietnam National Lung Hospital, Vietnam

\section{Hip tuberculosis at the stage IV with acetabular protrusion: A case report of diagnosis, medical and surgical treatment}

\author{
Hoan Do Dang, Trang Nguyen Khac and Nhung Nguyen Viet
}

DOI: $\underline{\text { https://doi.org/10.22271/27078345.2020.v2.i2a.20 }}$

\begin{abstract}
Tuberculosis osteoarthritis is an insidious disease. Up to now, due to contemptuous attitude, hip tuberculosis is easily mimic with other hip problems such as osteoarthritis, avascular necrosis or other bacterial infections. Besides, the diagnosis of hip tuberculosis requires specialized tests of the tuberculosis specialist, so the patients miss the opportunity for earlier treatment. The delay in TB treatment facilitates hip destruction leading to difficulty in hip reconstruction. We would like to report a special case of hip tuberculosis with difficult diagnosis, long-term medical treatment, and successful surgery of hip replacement.
\end{abstract}

Keywords: hip tuberculosis, hip replacement

\section{Introduction}

According to current estimates by the World Health Organization, the rate of tuberculosis infection are as high as one-third of the world's population ${ }^{[1]}$. Hip tuberculosis constitutes approximately $15 \%$ of all cases of osteoarticular tuberculosis ${ }^{[2]}$. Hip tuberculosis is often diagnosed late after excluding other infection of the hip, therefore patients may lose the opportunity to receive treatment earlier. Mycobacteria tuberculosis devastate hip joint in many ways. They may cause synovitis, start an inflammation reaction chain. They may form intra-articular abscesses, making an increasing in intra-articular pressure which lead to avascular necrosis later. They may destroy acetabular, head and neck of femur result in joint deformity and joint dislocation. According to S.K. Saraf et al. ${ }^{[2]}$, Early diagnosis and effective chemotherapy are vital to save the joint. Diagnostic procedures include capturing diagnostic imagines; taking out pus and necrotic tissues for tuberculosis histopathology, tuberculosis culture and tuberculosis gens detection ${ }^{[2]}$. Treatments of hip tuberculosis at stage IV remain controversial. In general, patients start with taking anti-tuberculosis drugs then experienced hip surgery. According to real conditions, the surgeon can choose one kind of surgery from excision arthroplasty, joint arthrodesis or joint replacement.

We report a patient with stage IV of left hip tuberculosis, who has a type IIIC destruction of acetabular according to Paprosky [3], abscesses formation both intra-articular and extraarticular. Treatment included left hip arthrotomy, anti-tuberculosis drugs as formula 2RHZE/10RHE. 2 months after hip arthrotomy he had left hip total replacement, using an anti-protrusion cage. On following up 24 months, patients had a good hip movement, mild pain, no recurrence of the abscesses.

\section{Case report}

A 28-year-old male patient came to our hospital in the month of January 2018 with complaints of pain in the left hip that he had since past 11 months and inability in bearing weight and walking that he had faced since past 3 months. The hip pain progressed more and more severe and made the patient intend to commit suicide.

The physical examination showed a left hip stiffness on flexion, adduction, and internal rotation position. The patient felt the worst pain even when lying in bed, hip score as Harris classification was 0 point ${ }^{[4]}$. On the AP X-rays and CT scan, the hip was seen as severe damage on the left. Femur head was dissipated, acetabular was collapsed as type IIIA following Paprosky classification ${ }^{[3]}$. X-ray of the chest was normal. On the magnetic resonance image, there was a heterogeneous para-articular abscess with a smooth rim which travel into ischio-rectal fossa on the left hip, join intra-articular abscess, bone marrow edema 
of the acetabular were both seen too. Serologically, he was not positive to HIV, HbsAg and HCV, his hemoglobin was low, ESR and CRP were grossly elevated and peripheral smear showed a normocytic hypochromic Fig., a decision was made to do left hip arthrocentesis and to send the aspirate for gram staining, Bactec culture and Genxpert MTB/RIF. After 3 days the Genxpert result came out to be positive and Bactec culture turned out to be positive 3 weeks later.

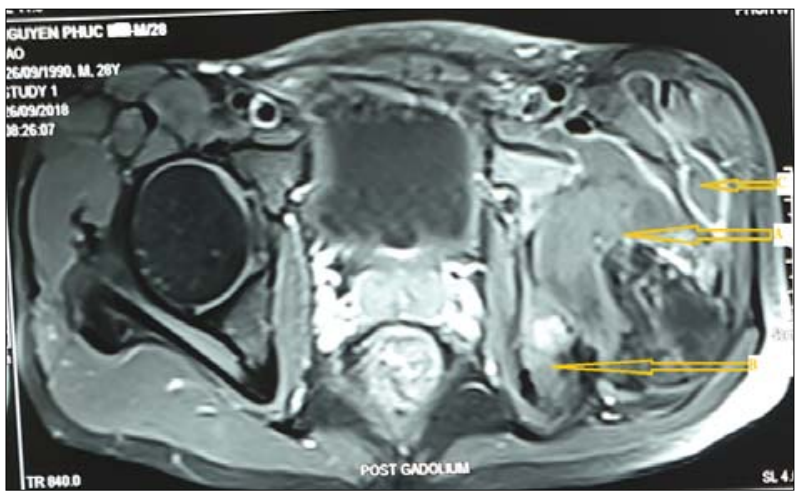

Fig 1: MRI showed intra-articular abscess (A); Para-articular abscess $(\mathrm{B}, \mathrm{C})$

The patient was treated with a combination of antituberculosis drugs following the formula 2RHZE/10RHE. Rifampicin, Isoniazid, Pyrazinamide and Ethambutol was taking daily in the first 2 months, then Rifampicin, Isoniazid and Ethambutol were taking daily in further 10 months. After 2 weeks of multidrug antitubercular medication, he was performed left hip arthrotomy and join debridement. After 2 months follow up, groin's pain decreased, VAS 4 points, patient' weight increased by $5 \mathrm{Kg}$, but, there was still limited in hip movement, hip function according to Harris classification was 29 points ${ }^{[4]}$, ultrasonography found no abscess in the joint, the blood test showed no anemia, CRP was at $8 \mathrm{mg} /$ dl. He had a total hip replacement done using an antiprotrusion cage without the need for additional bone grafting to reconstruct the acetabular. Postoperative, he restored to full range of hip motion. He started to walk with the help of a crutches frame by day 5 after surgery. After 24 months of postoperative follow-up, his left hip showed a very good function with a full range of motion, with no recurrence of the abscess, pain according to the VAS 1 point, hip function according to Harris classification 94 points.

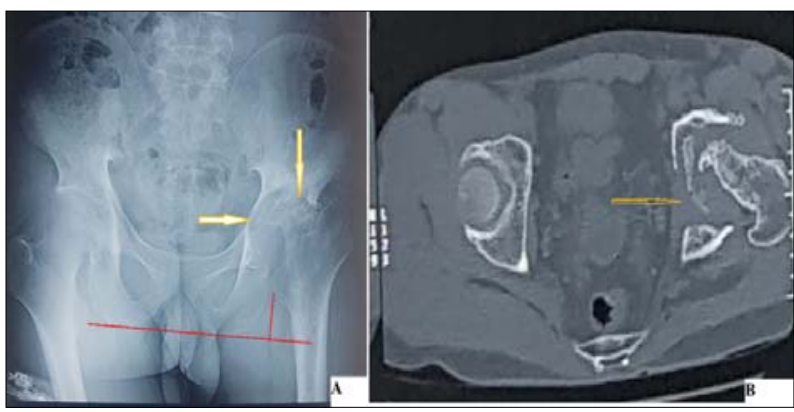

Fig 2: AP radiograph (A) and CT (B) showed a grossly bone destruction, dislocation of left hip before total hip replacement (The arrows indicate the areas of bone destruction)

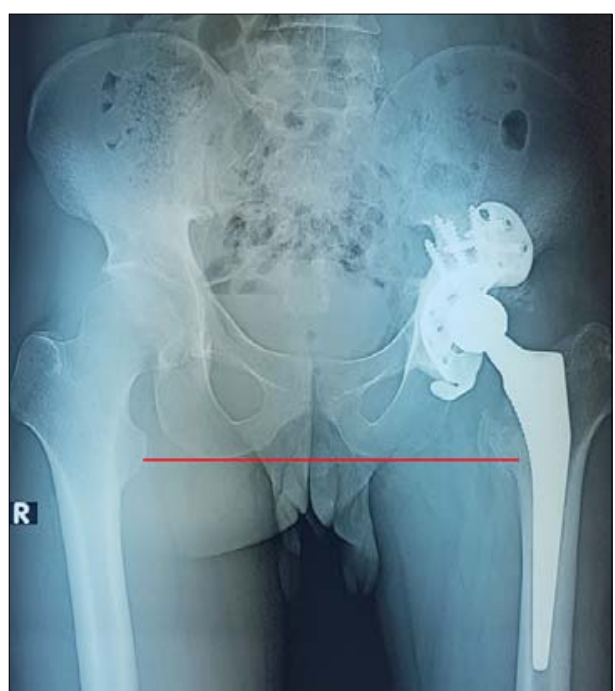

Fig 3: AP radiograph 2 year after total hip replacement

\section{Discussion}

Osteoarticular TB is secondary to primary pathology in lungs, lymph nodes or any of the viscera ${ }^{[5]}$. Through the hematogenous route, the bacteria reach either to synovium or bone. Osteoarticular manifestations may be intra-articular or extra-articular. When the disease starts as intra-articular, it progresses fast to involve the whole joint. Cold abscess that usually forms within the joint may perforate the capsule to present around the hip joint in the femoral triangle, medial, lateral or posterior aspects of the thigh, ischio-rectal fossa. The variable clinical and radiological presentations may mimic common osseous and articular conditions and they may delay the diagnosis of tuberculosis.

In 2002, Babhulkar and Pande ${ }^{[5]}$ introduced a classification of hip TB based on clinicoradiological presentations into 4 stages which include: Stage I: synovitis, stage II: early arthritis, stage III: arthritis and stage IV: advanced arthritis. In 2010, Tuli [2] suggested modification in this classification. He classified it as synovitis, early arthritis, advanced arthritis and advanced arthritis with subluxation/dislocation.

Stage IV of hip tuberculosis is characterized by advanced arthritis with subluxation or dislocation, severe damage to bone and joint construction. Clinically it presents outstanding pain, limb deformity and movement limiting. Pain is caused by many ways including deformation of the hip joint structure, inflammation triggered by the tuberculosis necrotic tissues causing pain by chains of reaction, intra-articular abscess increasing pressure in the joint to stimulate pain sensory nerve endings [6] Arthrotomy, debridement tuberculosis necrotic tissues resolve the problem of inflamed foci, simultaneously opens the anterior sac to lessen the pressure in the joint. However, for full restoring of hip structure in adult, total hip replacement (THR) surgery is the optimal choice ${ }^{[2]}$. Instead of controversial issues ${ }^{\text {[7] }}$ due to the worry of recurrence of tuberculosis and failure of join replacement, The acceptability of the THR is gradually increasing globally ${ }^{[8]}$. Babhulkar and Pande [5] recommended THR to be performed after 10 years or more between active infection and replacement surgery. Wang et al. [9] recommend a combination of antituberculosis drugs for at least 2 weeks prior to the operation and subsequently for at least 12 
months after the operation. Neogi et al. ${ }^{[10]}$ recommended a routine preoperative computed tomography-guided biopsy, with specimens subjected to culture and sensitivity testing, in view of the emergence of drug-resistant Tubercular bacilli. Sidhu et al. ${ }^{[11]}$ performed cemented THR in 23 patients with active tuberculous arthritis of the hip. His patients had received at least 3 months of antitubercular therapy before surgery and treatment were continued for a total of 18 months. With a mean follow-up of 4.7 years, no activation of the infection or implant-loosening was recorded. Lee et al. ${ }^{[12]}$ reported a two-stage surgery for patient with advanced active tuberculosis of the hip. Their protocol included the administration of antitubercular drugs for at least 2 weeks prior to the first surgery. At the first surgery, extensive debridement was considered and antibiotic-loaded cement spacer was used (Combination of streptomycin and vancomycin). Tuberculosis chemotherapy was continued for at least next four months. If C-reactive protein concentration and Erythrocyte sedimentation rate were normal, hip replacement was performed. The outcomes showed significant pain relief without any recurrence. In my opinion, I suppose that hip replacement could be done in active tuberculosis hip if the patient has all of the conditions followed:

1. Treatment with anti-tuberculosis drugs at least 2 weeks pre-operation with a proper formula.

2. There is no abscess formation intra-articular or paraarticular.

3. Joint's infection decrease to a stable condition that could be seen by serum C-reactive protein concentration lower than $10 \mathrm{mg} / \mathrm{dl}$; patient's weight gain.

However, reactive of TB is not the only problem in total hip replacement (THR) for tuberculosis coxitis. Stage IV tuberculosis is characterized by grossly bone destruction accompanied by dislocation. Bones loss often occur in the superior dome and medial wall causing dislocation ${ }^{[2]}$. If the acetabular is not reconstructed well, the THR may failure that is difficult to repair later ${ }^{[13]}$. Central hip dislocations ${ }^{[14]}$, femoral acetabular impingement ${ }^{[15]}$ after THR are some of the severe complications due to destruction of acetabular. In this case, acetabular was collapsed severely in the medial wall causing acetabular protrusion. We wondered to restore it with or without bone graft because bone graft could be a culture for tuberculosis reactivation. Fortunately, we found an intact area in patient's left pelvic wing so we can use an anti-protrusion cage ${ }^{[16]}$ without bone grafting to move bearing point to this area.

\section{Conclusion}

Hip tuberculosis is a disease that is difficult to diagnose and treat. Total hip replacement can be considered as the best treatment at the moment. However, attention should be paid to prevent reactive tuberculosis and complications due to grossly bone destruction.

\section{References}

1. World Health Organization. Global tuberculosis report, 2016. https://apps.who.int/iris/handle/10665/250441

2. Saraf SK, Tuli SM. Tuberculosis of hip: A current concept review. Indian journal of orthopaedics. 2015; 49(1):1-9.
3. Telleria JJM, Gee AO. Classifications in brief: Paprosky classification of acetabular bone loss. Clinical orthopaedics and related research. 2013; 471(11):37253730.

4. Nilsdotter A, Bremander A. Measures of hip function and symptoms: Harris Hip Score (HHS), Hip Disability and Osteoarthritis Outcome Score (HOOS), Oxford Hip Score (OHS), Lequesne Index of Severity for Osteoarthritis of the Hip (LISOH), and American Academy of Orthopedic Surgeons (AAOS) Hip and Knee Questionnaire. Arthritis Care \& Research. 2011; 63(S11):S200-S207.

5. Babhulkar S. Tuberculosis of the hip. Clin Orthop Relat Res. 2002; 398:93-9, 2002.

6. Tuli SM. Tuberculosis of the Skeletal System New Delhi: Jaypee Brothers Medical Publishers Pvt. Ltd. 2010; 4:69-110.

7. Parsa A et al. Hip Surgery in Quiescent or Active Tubercular Hip Arthritis; Is Reactivation Risk Really a Matter. The archives of bone and joint surgery. 2018; 6(3):169-175.

8. Tiwari A, Karkhur Y, Maini L. Total hip replacement in tuberculosis of hip: A systematic review. Journal of clinical orthopaedics and trauma. 2018; 9(1):54-57.

9. Wang Y. Total hip arthroplasty for active tuberculosis of the hip. Int Orthop. 2010; 34:1111-4.

10. Neogi DS, Ashok Kumar YC, Khan SA. Total hip arthroplasty in patients with active tuberculosis of the hip with advanced arthritis. Clin Orthop Relat Res. 2010; 468:605-12.

11. Sidhu AS, Singh AP. Total hip replacement in active advanced tuberculous arthritis. J Bone Joint Surg Br. 2009; 91:1301-4.

12. Li L et al. Two-stage total hip arthroplasty for patients with advanced active tuberculosis of the hip. Journal of orthopaedic surgery and research. 2016; 11:38-38.

13. Karachalios T, Komnos G, Koutalos A. Total hip arthroplasty: Survival and modes of failure. Effort open reviews. 2018; 3(5):232-239.

14. Dargel $\mathrm{J}$ et al. Dislocation following total hip replacement. Deutsches Arzteblatt international. 2014; 111(51-52):884-890.

15. Brown TD, Callaghan JJ. Impingement in Total Hip Replacement: Mechanisms and Consequences. Current orthopaedics. 2008; 22(6):376-391.

16. López-Torres II et al. Clinical and radiological outcomes of trabecular metal systems and antiprotrusion cages in acetabular revision surgery with severe defects: a comparative study. International Orthopaedics. 2018; 42(8):1811-1818. 